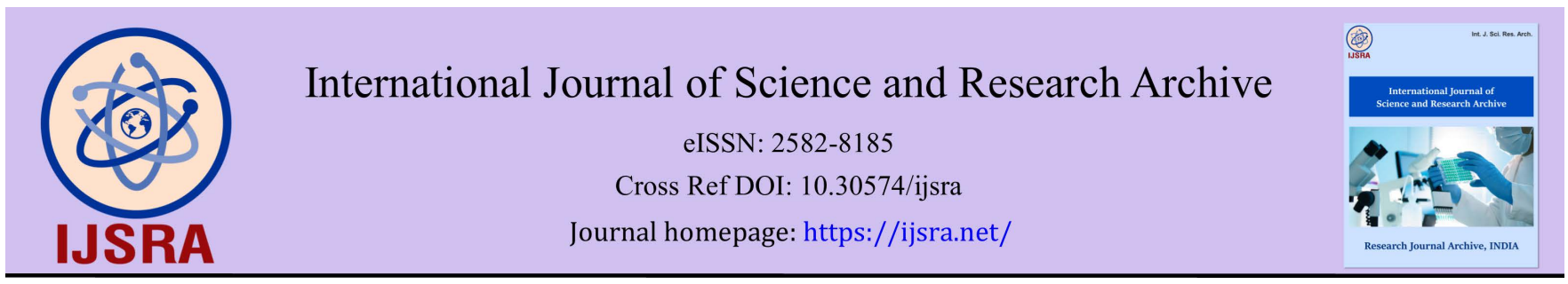

(CASE REPORT)

\title{
Pulsatile tinnitus as the first manifestation of a jugular paraganglioma
}

\author{
Athina L.Zarachi ${ }^{1,}{ }^{*}$, Angelos M.Liontos ${ }^{2}$, Ioannis D.Komnos ${ }^{1}$, Christina K.Naka ${ }^{3}$ and Ioannis G.Kastanioudakis ${ }^{1}$ \\ ${ }^{1}$ Department of Otorhinolaryngology, Head and Neck Surgery, Faculty of Medicine, School of Health Sciences, University \\ of Ioannina, Ioannina, Greece. \\ ${ }^{2}$ Faculty of Medicine, Department of Internal Medicine, School of Health Sciences, University of Ioannina, Ioannina, \\ Greece. \\ ${ }^{3}$ Faculty of Medicine, Department of Radiology, School of Health Sciences, University of Ioannina, Ioannina, Greece.
}

International Journal of Science and Research Archive, 2021, 03(02), 027-030

Publication history: Received on 08 August 2021; revised on 10 September 2021; accepted on 12 September 2021

Article DOI: https://doi.org/10.30574/ijsra.2021.3.2.0130

\begin{abstract}
We present the case of a 59 year old male patient with pulsate tinnitus, referred as the first manifestation of a jugular parganglioma. The patient examined at the outpatient ENT Department of our hospital with a left lateral cervical swelling, difficulty in swallowing and pulsatile tinnitus. Tinnitus was the first reported symptom that appeared one month before. The patient had no medical history. The clinical examination showed an asymmetric swelling of the left tonsil, a left vocal cord paralysis, a retrotympanic mass otoscopically and conductive hearing loss in the left ear. The patient underwent a head-and-neck computed tomography that raised the suspicion of a paraganglioma. The magnetic resonance imaging and the magnetic angiography with the administration of a contrast agent set the diagnosis of a jugular paraganglioma. After the tumor embolization, the patient unerwent primary resection of the tumor. This is the first case of a patient with a jugular paraganglioma and pulsatile tinnitus as the first reported symptom.
\end{abstract}

Keywords: Jugular paraganglioma; Pulsatile tinnitus; Embolization; First manifestation; Magnetic angiography; Glomus tumor

\section{Introduction}

Tinnitus refers to the sensation of audible sounds in the ear or head, with absence of external stimulation. They can be pulsating, that are due to an arterial or venous damage, or non-vascular. They can also be non-pulsating, that are due to a cochlear or a brain damage. Among patients with venous tinnitus, most of them appear sigmoid sinus diverticulum, carotid atherosclerotic disease, benign intracranial hypertension (BIH), high jugular bulb, transverse or sigmoid stenosis. Atherosclerotic disease carotid (ipsilateral or contralateral), dural arteriovenous fistulas, carotid-cavernous fistula, fibromuscular dysplasia of carotid artery, carotid artery dissection or hypertension are possible anomalies in patients with arterial pulsatile tinnitus. Non-vascular entities include superior semicircular canal dehiscence and benign intracranial hypertension. Pulsatile tinnitus requires a careful physical examination and evaluation with specific imaging techniques to identify the origin of the symptoms [1-5]. Glomus tumors, that are capable causes of pulsate tinnitus, originate from special neural crest elements, called paraganglion cells. Paragangliomas of the head and neck are vascular neoplasms and the site of origin defines the name given of these tumors. In the head and neck, they can commonly occur at the carotid bifurcation, the jugular bulb, the tympanic plexus on the promontory and the vagal nerve. Although in many patients with tympanic paraganglioma and jugular paraganglioma (JP), tinnitus and hearing loss can be early symptoms, patients with JP often suffer from lower cranial nerve deficits[6, 7]. We present the case of a patient with jugular paraganglioma that appeared pulsate tinnitus, as a first symptom.

\footnotetext{
* Corresponding author: Athina Zarachi

Department of Otorhinolaryngology, Head and Neck Surgery, Faculty of Medicine, School of Health Sciences, University of Ioannina, Ioannina, Greece.
}

Copyright $(2021$ Author(s) retain the copyright of this article. This article is published under the terms of the Creative Commons Attribution Liscense 4.0. 


\section{Case report}

A 59-year-old male patient examined in the outpatient clinic of ENT Department in our hospital, because of a left lateral cervical swelling, difficulty in swallowing and pulsatile tinnitus in the left ear, for about one month. According to the patient's medical history, he did not receive any medication. medicine. Patient reported that the first symptom he manifested was pulsatile tinnitus, followed by the other symptoms. The clinical examination of the oropharynx showed an asymmetric swelling of the left tonsil. Through video laryngoscopy a left vocal cord paralysis was diagnosed. A retrotympanic mass shown as a red shadow in the lower left part of the tympanic membrane, was detected otoscopically (Figure 1).

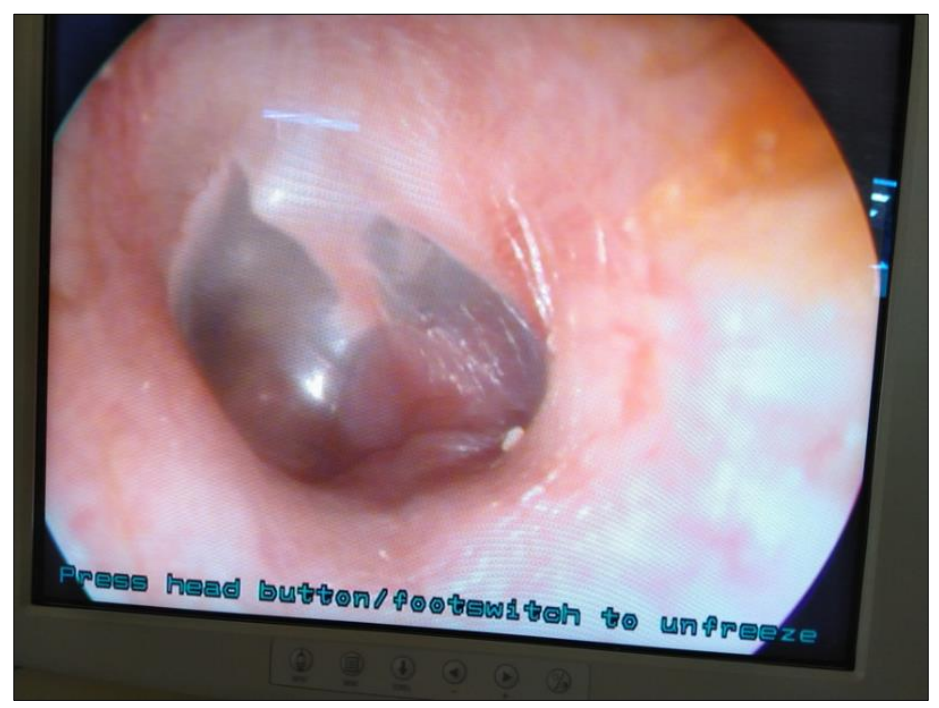

Figure 1 Ear endoscopic image shows a retrotympanic mass shown as a red shadow in the lower left part of the tympanic membrane

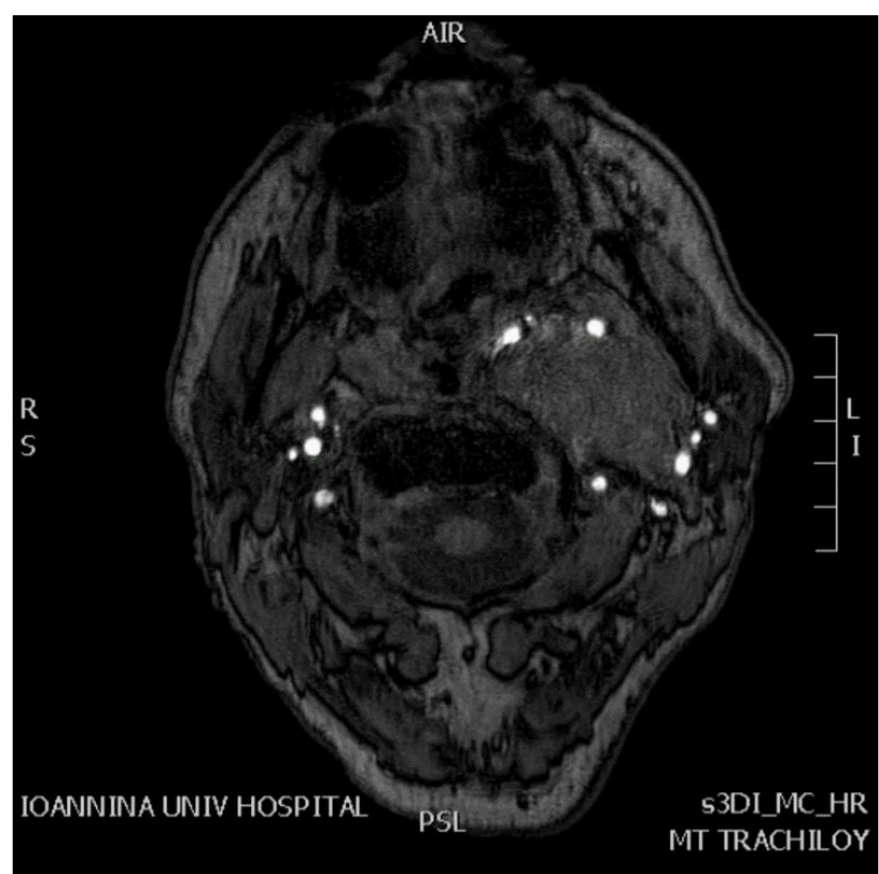

Figure 2 Magnetic resonance imaging shows a large lesion along the left carotid system that raises the suspicion of paraganglioma

The audiogram confirmed the conductive hearing loss in the left ear that was diagnosed through the examination with 512-Hertz tuning folk. The patient underwent a head-and-neck computed tomography that showed up a large lesion 
along the left carotid system that raises the suspicion of paraganglioma. The magnetic resonance imaging came to similar conclusions (Figure 2). A magnetic angiography (MA) with the administration of a contrast agent set the diagnosis. The MA showed a large mass measuring 29 × 48 × $96 \mathrm{~mm}$, extending from the scaphoid bulb to the lower part of the carotid division and reaching up to the posterior cranial fossa. This image was compatible with a paraganglioma protruding from the jugular bulb (Figure 3). The patient's therapeutic possibilities were discussed. The patient chose to address to a special endovascular embolization center, so to undergo vascular tumor embolization. After embolization the tumor underwent primary resection. We have no further information about the outcome of the patients' therapy, as he did not address our hospital again.

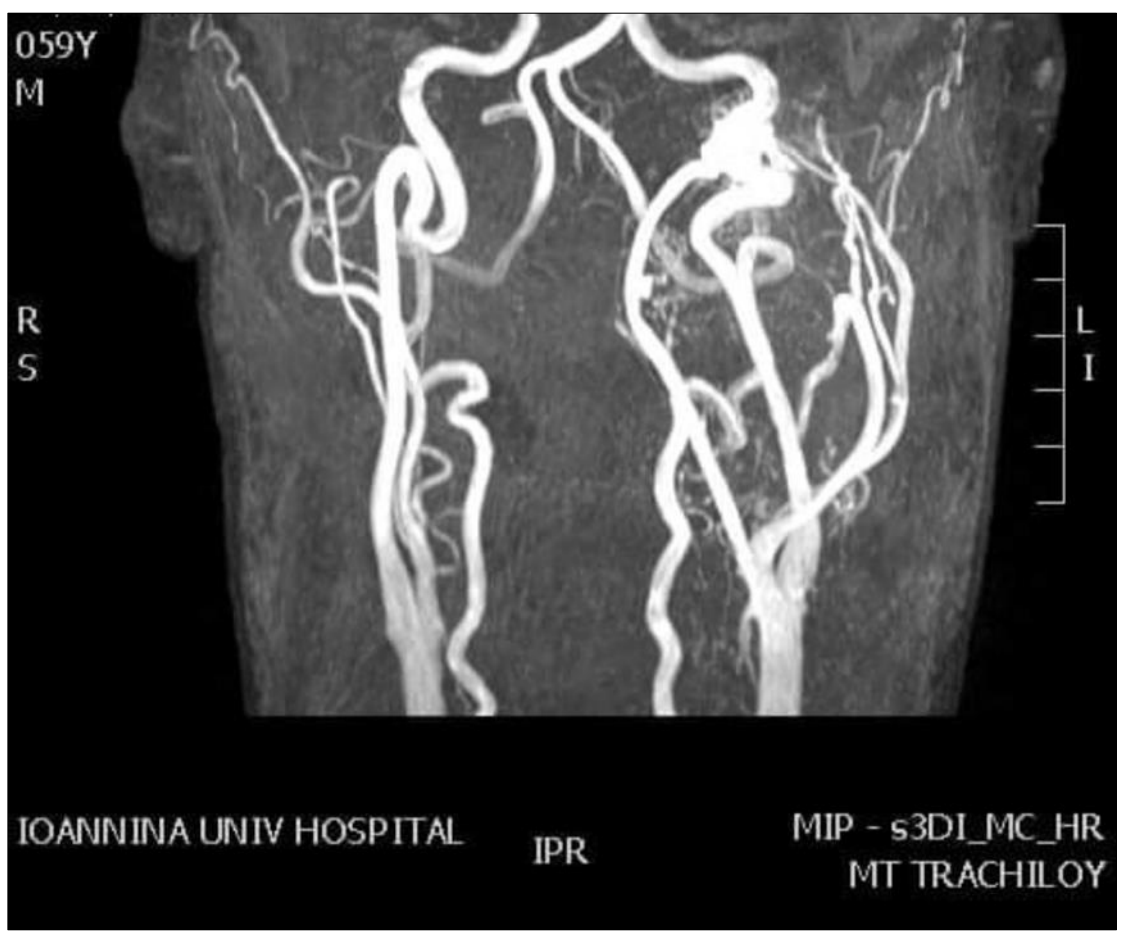

Figure 3 Magnetic angiography - contrast agent: a large mass measuring 29 x 48 x 96 mm, extending from the scaphoid bulb to the lower part of the carotid division and reaching up to the posterior cranial fossa. This image was compatible with a jugular paraganglioma

\section{Discussion}

Glomus jugul are tumors are fascinating lesions that arise from paraganglia cells situated near the jugular foramen. They associate with the lower cranial nerves and inner ear, so when they grow up they cause morbidity in these structures [8] . Tumors more commonly present with conductive hearing loss, pulsatile tinnitus, or lower cranial neuropathies due to mass effect and rarely they lead to cardiovascular effects such as tachycardia and labile blood pressure. Complete surgical excision of these highly haemorrhagic tumors is seldom possible because of their vascularity, invasive character, critical location, and often because of the advanced stage of the tumor at presentation [9-11] .

\section{Conclusion}

Pulsating tinnitus may indicate vascular disease and, less frequently due to glomus jugular paraganglioma. Among the symptoms that the JP can causes is pulsatile tinnitus. In our knowledge, this is the first case of a patient with a jugular paraganglioma and pulsate tinnitus, as the first reported symptom.

\section{Compliance with ethical standards}

\section{Disclosure of conflict of interest}

The authors declare no conflicts of interest regarding the publication of this paper. 


\section{Statement of informed consent}

Informed consent was obtained from all individual participants included in the study.

\section{References}

[1] Hofmann E, Behr R, Neumann-Haefelin T, Schwager K. Pulsatile Tinnitus. Deutsches Aerzteblatt Online. 2013.

[2] Mattox DE, Hudgins P.Algorithm for evaluation of pulsatile tinnitus. Acta Oto-Laryngologica. 2008; 128(4): 427431.

[3] Sismanis A. Pulsatile tinnitus. Otolaryngologic Clinics of North America. 2003; 36(2): 389-402.

[4] Sonmez G, Basekim CC, Ozturk E, Gungor A, Kizilkaya E. Imaging of pulsatile tinnitus: a review of 74 patients. Clinical Imaging. 2007; 31(2): 102-108.

[5] House WF, Glasscock ME. Glomus Tympanicum Tumors. Archives of Otolaryngology - Head and Neck Surgery. 1968; 87(5): 550-554.

[6] Jackson CG. Glomus tympanicum and glomus jugulare tumors. Otolaryngologic Clinics of North America. 2001; 34(5): 941-970.

[7] Boedeker CC, Ridder GJ, Schipper J. Paragangliomas of the head and neck: diagnosis and treatment. Familial Cance. 2005; 4(1): 55-59.

[8] Heth J. The basic science of glomus jugulare tumors. Neurosurgical Focus. 2004; 17(2): 6-11.

[9] Al-Mefty 0, Teixeira A. Complex tumors of the glomus jugulare: criteria, treatment, and outcome. Neurosurgical Focus.. 2004; 17(2): 1356-1366.

[10] Tran Ba Huy P. Radiotherapy for glomus jugulare paraganglioma. European Annals of Otorhinolaryngology, Head and Neck Diseases. 2014; 131(4): 223-226.

[11] Shapiro S, Kellermeyer B, Ramadan J, Jones G, Wiseman B, Cassis A. Outcomes of Primary Radiosurgery Treatment of Glomus Jugulare Tumors. Otology \& Neurotology. 2018; 1. 